\title{
A Homogeneous Y-branch Type Polarization Splitter With Electrically-Tunable Output Powers on Lithium Niobate
}

\author{
Wen-Hao Hsu, Ko-Chun Lin, and Way-Seen Wang \\ Department of Electrical Engineering and Graduate Institute of Electro-Optical Engineering \\ National Taiwan University, Taipei 10617,Taiwan,ROC Email: wswang@cc.ee.ntu.edu.tw
}

\begin{abstract}
Polarization splitters are key cormponents in coherent detection and polarization-diversity circuits. The output powers of the polarization splitter usually fluctuate with time or deviate from the optimal design such as 50:50 output power ratio, and the circuit performance will be degraded. To overcome this problem, a tuning mechanism is needed to stabilize or compensate the output powers of polarization splitter. In this work (NSC91-2215-E-002-024), we demonstrate a tunable polarization splitter, in which the output powers of transverse electric (TE) and transverse magnetic (TM) modes at two arms can be electrically tuned by integrating a TE-TM mode converter [1] with the polarization splitter. To reduce the complexity of design and fabrication, a homogenous Y-branch structure made by only zinc and nickel co-diffusion into lithium niobate $\left(\mathrm{LiNbO}_{3}\right)$ is used for the polarization splitter. Neither combination of different fabrication processes nor special design for the waveguides dimensions is required. That is due to the guided polarization of zinc and nickel co-diffused $\mathrm{LiNbO}_{3}$ waveguide can be changed by using different fabrication parameters [2].
\end{abstract}

The proposed tunable polarization splitter is shown in Fig. 1. The waveguide width $w$ is $8 \mu \mathrm{m}$ and the branch angle $\theta$ is $0.5^{\circ}$. First, a zinc strip of thickness $750 \AA$ and a nickel strip of thickness $200 \AA$ are deposited as the input $(\# 1)$ and the TE-output waveguide (\#2). Then another zinc strip of thickness $1000 \AA$ is added on the input waveguide for it takes more zinc to form a randomly polarized waveguide under the same diffusion condition. The strips are then diffused at $950^{\circ} \mathrm{C}$ for $1.5 \mathrm{hr}$ to form the input and TE-output waveguides. The same process is repeated again to form the TM-output waveguide (\#3) except at a lower temperature $800^{\circ} \mathrm{C}$. Note that though the input and TE-output waveguides are diffused twice, no significant influence on the guiding property was found. After the polarization splitter is accomplished, a pair of electrodes is fabricated around the input waveguide for TE-TM mode conversion via the use of electro-optic coefficient $r_{51}$. The electrodes have to be periodic because of the birefringence of $z$-cut $\mathrm{LiNbO}_{3}$. The period is determined by the phase matching condition $(2 \pi / \lambda)\left|n_{\mathrm{TE}}-n_{\mathrm{TM}}\right|=(2 \pi / \Lambda)$, where $\lambda$ is the laser wavelength and $\Lambda$ is the electrode period. For $\mathrm{LiNbO}_{3},\left|n_{\mathrm{TE}}-n_{\mathrm{TM}}\right|=0.0734$ at $\lambda=1.55 \mu \mathrm{m}$, and $\Lambda$ is calculated to be $21 \mu \mathrm{m}$. The electrodes, fabricated by etching in $85 \%$ phosphorous acid at $70^{\circ} \mathrm{C}$ for $20 \mathrm{sec}$, are $4-\mathrm{mm}$ long with a gap of $12 \mu \mathrm{m}$.

The output power of each mode is captured with a charge-coupled device camera. The field contour profiles show better mode matching is observed as compared to that uses heterogeneous structure [1]. The extinction ratios for TE and TM mode are $23 \mathrm{~dB}$ and $18 \mathrm{~dB}$, respectively. To demonstrate the tunability of the polarization splitter, a tunable voltage $V$ app was applied to the electrodes, and the output powers of both arms were measured as shown in Fig. 2 . As can be seen, when $V$ app $=100 \mathrm{~V}, 40 \%$ of the output power at one arm is converted and directed to the other arm. With the same laser wavelength, electrode gap, and electrode length, the estimated voltage for a $40 \%$ mode conversion is compatible to that reported [2]. Existing methods such as depositing longer electrodes or etching ridge structure near the input waveguide can be used to reduce the tuning voltage significantly. For example, with the aid of a ridge of height $2 \mu \mathrm{m}$ can have a tunability of about $50 \%$ when $V_{\text {app }}=70 \mathrm{~V}$. Details of the improvement and application of the proposed device will be of great interests in the future.

[1] J. Y. Li, W. H. Hsu, and W. S. Wang, CLEO/Pacific Rim 2001, vol. 1, pp. 94-95, 2001.

[2] R. C. Alferness, Appl. Phys. Lett., vol. 36, no. 7, pp. 513-515, 1980. 